\title{
An Asymptotic Formula for $r$-Bell Numbers with Real Arguments
}

\author{
Cristina B. Corcino ${ }^{1}$ and Roberto B. Corcino ${ }^{2}$ \\ ${ }^{1}$ Institute of Mathematics, University of the Philippines, Diliman, Quezon City 1004, Philippines \\ ${ }^{2}$ Department of Mathematics, Mindanao State University, Marawi City 9700, Philippines
}

Correspondence should be addressed to Roberto B. Corcino; rcorcino@yahoo.com

Received 24 December 2012; Accepted 15 January 2013

Academic Editors: A. Ashrafi and H. Deng

Copyright (C) 2013 C. B. Corcino and R. B. Corcino. This is an open access article distributed under the Creative Commons Attribution License, which permits unrestricted use, distribution, and reproduction in any medium, provided the original work is properly cited.

The $r$-Bell numbers are generalized using the concept of the Hankel contour. Some properties parallel to those of the ordinary Bell numbers are established. Moreover, an asymptotic approximation for $r$-Bell numbers with real arguments is obtained.

\section{Introduction}

The $r$-Stirling numbers of the second kind, denoted by $\left\{\begin{array}{l}n \\ k\end{array}\right\}_{r}$, are defined by Broder in [1], combinatorially, to be the number of partitions of the set $\{1,2, \ldots, n\}$ into $k$ nonempty subsets, such that the numbers $1,2, \ldots, r$ are in distinct subsets. Several properties of these numbers are established in [1-3]. Further generalization was established in [4] which is called $(r, \beta)$-Stirling numbers. These numbers are equivalent to the $r$-Whitney numbers of the second kind [5] and the Rucinski-Voigt numbers [6].

The sum of $r$-Stirling numbers of the second kind for integral arguments was first considered by Corcino in [7] and was called the $r$-Bell numbers. Corcino obtained an asymptotic approximation of $r$-Bell numbers using the method of Moser and Wyman. Here, we use $B_{n, r}$ to denote the $r$-Bell numbers; that is,

$$
B_{n, r}=\sum_{k=0}^{n}\left\{\begin{array}{l}
n+r \\
k+r
\end{array}\right\}_{r}
$$

In a followup study of Mezö [8], the $r$-Bell numbers $B_{n, r}$ were given more properties. One of these is the following exponential generating function:

$$
\sum_{n \geq 0} B_{n, r} \frac{t^{n}}{n !}=\exp \left(r t+\left(e^{t}-1\right)\right)
$$

A more general form of Bell numbers, denoted by $G_{x, r, \beta}$, was defined in [9] as

$$
G_{x, r, \beta}=\frac{x !}{2 \pi i} \int_{\mathscr{H}} e^{r z+\left(e^{\beta z}-1\right) / \beta} \frac{d z}{z^{x+1}},
$$

where the parameters $x, r$, and $\beta$ are complex numbers with $\mathbb{R} r>0, \mathbb{R} \beta>0$, and $x !=\Gamma(x+1)$. In this paper, we define the $r$-Bell numbers with complex argument using the concept of Hankel contour and establish some properties parallel to those obtained by Mező in [8]. Moreover, an asymptotic formula of these numbers for real arguments will be derived using the method of Moser and Wyman [10].

\section{2. $r$-Stirling Numbers of the Second Kind}

Graham et al. [11] proposed another way of generalizing the Stirling numbers by extending the range of values of the parameters $n$ and $k$ to complex numbers. This problem was first considered by Flajolet and Prodinger [12] by defining the classical Stirling numbers with complex arguments using the concept of Hankel contour. Recently, the $(r, \beta)$-Stirling numbers with complex arguments, denoted by $\left\{\begin{array}{l}x \\ y\end{array}\right\}_{\beta, r}$, were defined in [9] by means of the following integral representation over a Hankel contour $\mathscr{H}$ :

$$
\left\{\begin{array}{l}
x \\
y
\end{array}\right\}_{\beta, r}=\frac{x !}{y ! \beta^{y} 2 \pi i} \int_{\mathscr{C}} e^{r z}\left(e^{\beta z}-1\right)^{y} \frac{d z}{z^{x+1}},
$$

where $r$ and $\beta$ are complex numbers with $\mathbb{R} r>0, \mathbb{R} \beta>0$, and $x !=\Gamma(x+1)$. We know that, for integral case, the $r$-Stirling 
numbers of the second kind may be obtained by taking $\beta=$ 1 . Hence, using (4), we can define the second-kind $r$-Stirling numbers with complex arguments as follows.

Definition 1. The $r$-Stirling numbers of the second kind $\left\{\begin{array}{l}x+r \\ y+r\end{array}\right\}_{r}$ of complex arguments $x$ and $y$ are defined by

$$
\left\{\begin{array}{l}
x+r \\
y+r
\end{array}\right\}_{r}:=\frac{x !}{y ! 2 \pi i} \int_{\mathscr{C}} e^{r z}\left(e^{z}-1\right)^{y} \frac{d z}{z^{x+1}},
$$

where $r$ is complex number with $\mathbb{R} r>0$ and $x !=\Gamma(x+1)$, and the logarithm involved in the functions $\left(e^{z}-1\right)^{y}$ and $z^{x+1}$ is taken to be the principal branch. The Hankel contour $\mathscr{H}$ starts from $-\infty$ below the negative axis, surrounds the origin counterclockwise, and returns to $-\infty$ in the half plane $\mathbb{I} z>0$, such that it has a distance $\epsilon \leq 1$ from the nonpositive real axis.

Remark 2. Since $\mathbb{R} r>0$ and $\mathbb{R} z \rightarrow-\infty$, the integral in (5) converges for all values of $x$ and $y$. Also, $\left\{\begin{array}{c}x+r \\ y+r\end{array}\right\}_{r}$ is a meromorphic function of $x$ (for any fixed $y$ ) with poles at the nonpositive integers, and it is entire as a function of $y$ (for any fixed $x$ not a negative integer).

Remark 3. By the change of variable on the integral in (4), say $w=\beta z$, we can express $\left\{\begin{array}{l}x \\ y\end{array}\right\}_{\beta, r}$ in terms of $\left\{\begin{array}{l}x+r \\ y+r\end{array}\right\}_{r}$ as follows:

$$
\left\{\begin{array}{l}
x \\
y
\end{array}\right\}_{\beta, r}=\beta^{x-y}\left\{\begin{array}{c}
x+\frac{r}{\beta} \\
y+\frac{r}{\beta}
\end{array}\right\}_{r / \beta} .
$$

Because of relation (6), every property of $(r, \beta)$-Stirling numbers with complex arguments will have a corresponding property for $r$-Stirling numbers with complex arguments and vice versa. For instance, the $(r, \beta)$-Stirling numbers in [9] satisfy the following relation:

$$
\left\{\begin{array}{l}
x \\
y
\end{array}\right\}_{\beta, r}=\left\{\begin{array}{l}
x-1 \\
y-1
\end{array}\right\}_{\beta, r}+(\beta y+r)\left\{\begin{array}{c}
x-1 \\
y
\end{array}\right\}_{\beta, r} .
$$

Replacing $r$ with $\beta r$, we get

$$
\left\{\begin{array}{l}
x \\
y
\end{array}\right\}_{\beta, \beta r}=\left\{\begin{array}{l}
x-1 \\
y-1
\end{array}\right\}_{\beta, \beta r}+(\beta y+\beta r)\left\{\begin{array}{c}
x-1 \\
y
\end{array}\right\}_{\beta, \beta r} .
$$

Using (6), we obtain

$$
\begin{aligned}
& \beta^{x-y}\left\{\begin{array}{c}
x+\frac{\beta r}{\beta} \\
y+\frac{\beta r}{\beta}
\end{array}\right\}_{\beta r / \beta} \\
& =\beta^{x-y}\left\{\begin{array}{l}
x+\left(\frac{\beta r}{\beta}\right)-1 \\
y+\left(\frac{\beta r}{\beta}\right)-1
\end{array}\right\}_{\beta r / \beta}
\end{aligned}
$$

$$
+\beta^{x-1-y}(\beta y+\beta r)\left\{\begin{array}{c}
x+\left(\frac{\beta r}{\beta}\right)-1 \\
y+\beta r
\end{array}\right\}_{\beta r / \beta} .
$$

Thus, we have

$$
\left\{\begin{array}{l}
x+r \\
y+r
\end{array}\right\}_{r}=\left\{\begin{array}{l}
x+r-1 \\
y+r-1
\end{array}\right\}_{r}+(y+r)\left\{\begin{array}{c}
x+r-1 \\
y+r
\end{array}\right\}_{r} .
$$

On the other hand, the $(r, \beta)$-Stirling numbers in [9] satisfy the relation

$$
\left\{\begin{array}{l}
x \\
y
\end{array}\right\}_{\beta, r}=\frac{1}{\beta^{y} y !} \sum_{j=0}^{\infty}(-1)^{y-j}\left(\begin{array}{l}
y \\
j
\end{array}\right)(\beta j+r)^{x} .
$$

Again, replacing $r$ with $\beta r$, we obtain

$$
\left\{\begin{array}{l}
x \\
y
\end{array}\right\}_{\beta, \beta r}=\frac{1}{\beta^{y} y !} \sum_{j=0}^{\infty}(-1)^{y-j}\left(\begin{array}{l}
y \\
j
\end{array}\right)(\beta j+\beta r)^{x} .
$$

By (6), we get

$$
\beta^{x-y}\left\{\begin{array}{c}
x+\frac{\beta r}{\beta} \\
y+\frac{\beta r}{\beta}
\end{array}\right\}_{\beta r / \beta}=\frac{1}{\beta^{y} y !} \sum_{j=0}^{\infty}(-1)^{y-j}\left(\begin{array}{c}
y \\
j
\end{array}\right)(\beta j+\beta r)^{x} .
$$

Thus, we have

$$
\left\{\begin{array}{l}
x+r \\
y+r
\end{array}\right\}_{r}=\frac{1}{y !} \sum_{j=0}^{\infty}(-1)^{y-j}\left(\begin{array}{l}
y \\
j
\end{array}\right)(j+r)^{x} .
$$

Let's state these relations formally in the following theorem.

Theorem 4. The r-Stirling numbers of the second kind with complex arguments satisfy the following relations:

$$
\begin{gathered}
\left\{\begin{array}{l}
x+r \\
y+r
\end{array}\right\}_{r}=\left\{\begin{array}{l}
x+r-1 \\
y+r-1
\end{array}\right\}_{r}+(y+r)\left\{\begin{array}{c}
x+r-1 \\
y+r
\end{array}\right\}_{r}, \\
\left\{\begin{array}{l}
x+r \\
y+r
\end{array}\right\}_{r}=\frac{1}{y !} \sum_{j=0}^{\infty}(-1)^{y-j}\left(\begin{array}{l}
y \\
j
\end{array}\right)(j+r)^{x} .
\end{gathered}
$$

Remark 5. We can give an alternative proof of this theorem. That is, by applying integration by parts on (5) and using the fact that $e^{\beta z}\left(e^{z}-1\right)^{y-1}=\left(e^{z}-1\right)^{y}+\left(e^{z}-1\right)^{y-1}$, we obtain

$$
\begin{aligned}
\left\{\begin{array}{l}
x+r \\
y+r
\end{array}\right\}_{r}= & \frac{(x-1) !}{(y-1) ! 2 \pi i} \int_{\mathscr{H}} e^{r z}\left(e^{z}-1\right)^{y-1} \frac{d z}{z^{x}} \\
& +\frac{(y+r)(x-1) !}{y ! 2 \pi i} \int_{\mathscr{H}} e^{r z}\left(e^{z}-1\right)^{y} \frac{d z}{z^{x}} .
\end{aligned}
$$


This implies (15) immediately. On the other hand, using Newton's Binomial Theorem, (5) can be expressed as

$$
\begin{aligned}
\left\{\begin{array}{c}
x+r \\
y+r
\end{array}\right\}_{r} & =\frac{x !}{y !} \frac{1}{2 \pi i} \int_{\mathscr{H}} e^{r z} \sum_{j=0}^{\infty}(-1)^{y-j}\left(\begin{array}{c}
y \\
j
\end{array}\right) e^{\beta z j} \frac{d z}{z^{x+1}} \\
& =\frac{1}{y !} \sum_{j=0}^{\infty}(-1)^{y-j}\left(\begin{array}{c}
y \\
j
\end{array}\right) \frac{x !}{2 \pi i} \int_{\mathscr{H}} e^{(j+r) z} \frac{d z}{z^{x+1}} .
\end{aligned}
$$

We know that Hankel's contour integral is a unit for gamma function over the set of complex numbers; that is,

$$
\left(\frac{i}{2 \pi} \int_{\mathscr{H}}(-z)^{-x} e^{-z} d z\right) \Gamma(x)=1, \quad|z|<\infty .
$$

This implies that

$$
\frac{x !}{2 \pi i} \int_{\mathscr{H}} e^{(j+r) z} \frac{d z}{z^{x+1}}=(j+r)^{x}
$$

This completes the proof of (16).

The next theorem contains a property for $\left\{\begin{array}{l}x+r \\ y+r\end{array}\right\}_{r}$ which is analogous to the identity that usually defines Stirling-type numbers.

Theorem 6. The r-Stirling numbers of the second kind with complex arguments satisfy the following relation:

$$
(t+r)^{x}=\sum_{k=0}^{\infty}\left\{\begin{array}{l}
x+r \\
k+r
\end{array}\right\}_{r}(t)_{k}
$$

where $(t)_{k}=t(t-1) \cdots(t-k+1)$.

Proof. Using (5) with $y=k$, a nonnegative integer, we obtain

$$
\begin{aligned}
\sum_{k=0}^{\infty}\left\{\begin{array}{l}
x+r \\
k+r
\end{array}\right\}_{r}(t)_{k} & =\sum_{k=0}^{\infty}\left\{\frac{x !}{k ! 2 \pi i} \int_{\mathscr{H}} e^{r z}\left(e^{z}-1\right)^{k} \frac{d z}{z^{x+1}}\right\}(t)_{k} \\
& =\frac{x !}{2 \pi i} \int_{\mathscr{C}} e^{r z}\left\{\sum_{k=0}^{\infty}\left(\begin{array}{l}
t \\
k
\end{array}\right)\left(e^{z}-1\right)^{k}\right\} \frac{d z}{z^{x+1}} \\
& =\frac{x !}{2 \pi i} \int_{\mathscr{H}} e^{r z}\left(e^{z}-1+1\right)^{t} \frac{d z}{z^{x+1}} \\
& =\frac{x !}{2 \pi i} \int_{\mathscr{H}} e^{r z} e^{t z} \frac{d z}{z^{x+1}} .
\end{aligned}
$$

Thus, by (20), we prove the theorem.

Remark 7. It is worth mentioning that this type of property was not established for $(r, \beta)$-Stirling numbers with complex arguments. However, replacing $t$ with $\beta^{-1} t$ and $r$ with $r / \beta$ in (21), we obtain

$$
\begin{aligned}
\left(\beta^{-1} t+\frac{r}{\beta}\right)^{x} & =\sum_{k=0}^{\infty}\left\{\begin{array}{c}
x+\frac{r}{\beta} \\
k+\frac{r}{\beta}
\end{array}\right\}_{r / \beta}\left(\beta^{-1} t\right)_{k}, \\
(t+r)^{x} & =\sum_{k=0}^{\infty} \beta^{x-k}\left\{\begin{array}{l}
x+\frac{r}{\beta} \\
k+\frac{r}{\beta}
\end{array}\right\}_{r / \beta}(t \mid \beta)_{k} .
\end{aligned}
$$

Using relation (6), we get

$$
(t+r)^{x}=\sum_{k=0}^{\infty}\left\{\begin{array}{l}
x \\
k
\end{array}\right\}_{\beta, r}(t \mid \beta)_{k} .
$$

This is the corresponding property for $(r, \beta)$-Stirling numbers with complex arguments.

Remark 8. In a separate paper of the present authors, an asymptotic formula for $r$-Stirling numbers of the second kind with real arguments was established using the method of Chelluri et al. [13].

\section{3. $r$-Bell Numbers}

Using Cauchy's integral formula, (2) can be transformed into the following integral representation of $B_{n, r}$ :

$$
B_{n, r}=\frac{n !}{2 \pi i} \int_{\gamma} e^{r z+\left(e^{z}-1\right)} \frac{d z}{z^{n+1}},
$$

where the integral contour $\gamma$ is a small contour encircling the origin. Since $n$ is nonnegative in (25), the contour $\gamma$ can be deformed into a Hankel contour $\mathscr{H}$ that starts from $-\infty$ below the negative axis, surrounds the origin counterclockwise, and returns to $-\infty$ in the half plane $\mathbb{I} z>0$. We assume that it is at distance $\leq 1$ from the real axis. Now, let us consider the following definition for the generalization of $r$-Bell numbers $B_{n, r}$ where $n$ is a complex number.

Definition 9. The $r$-Bell numbers $B_{x, r}$ of complex argument $x$ are defined by

$$
B_{x, r}=\frac{x !}{2 \pi i} \int_{\mathscr{\ell}} e^{r z+\left(e^{z}-1\right)} \frac{d z}{z^{x+1}},
$$

where $r$ is complex numbers with $\mathbb{R} r>0$ and $x !=\Gamma(x+1)$.

Since $\mathbb{R} r>0$ and $\mathbb{R} z \rightarrow-\infty$, clearly, the integral in (26) converges for all values of $x$. Moreover, $B_{x, r}$ is a meromorphic function of $x$ with poles at the nonpositive integers.

It can easily be shown that

$$
B_{x, r}=\sum_{k=0}^{\infty}\left\{\frac{1}{k !} \sum_{j=0}^{k}(-1)^{k-j}\left(\begin{array}{c}
k \\
j
\end{array}\right) \frac{x !}{2 \pi i} \int_{\mathscr{H}} e^{(j+r) z} z^{-(x+1)} d z\right\} .
$$


By (16) and (20), we obtain

$$
B_{x, r}=\sum_{k=0}^{\infty}\left\{\frac{1}{k !} \sum_{j=0}^{k}(-1)^{k-j}\left(\begin{array}{l}
k \\
j
\end{array}\right)(j+r)^{x}\right\}=\sum_{k=0}^{\infty}\left\{\begin{array}{l}
x+r \\
k+r
\end{array}\right\}_{r} .
$$

Hence, we have the following theorem.

Theorem 10. The r-Bell numbers are equal to

$$
B_{x, r}=\sum_{k=0}^{\infty}\left\{\begin{array}{l}
x+r \\
k+r
\end{array}\right\}_{r}
$$

where $x$ and $r$ are complex numbers.

By Theorem 10, we have verified that identity in (1) also holds for $B_{x, r}$ with complex argument $x$. This means that the numbers $B_{x, r}$ in Definition 9 belong to the family of Bell numbers.

The next theorem asserts that $B_{x, r}$ also possess a kind of Dobiński's formula which can easily be shown using Definiton 9 and the expansion of $e^{\left(r z+e^{z}-1\right)}$.

Theorem 11. The r-Bell numbers are equal to

$$
B_{x, r}=\frac{1}{e} \sum_{k=0}^{\infty} \frac{(k+r)^{x}}{k !}
$$

where $x$ and $r$ are complex numbers.

Using (26) and (30), we obtain the following corollary which is a kind of extension of the integral formula obtained by Mezö in [8].

Corollary 12. The following integral identity holds:

$$
\sum_{k=0}^{\infty} \frac{(k+r)^{x}}{k !}=\frac{x ! e}{2 \pi i} \int_{\mathscr{H}} e^{r z+\left(e^{z}-1\right)} \frac{d z}{z^{x+1}} .
$$

The $r$-Bell polynomials of Mezö [8] satisfy the recurrence relation

$$
B_{x, r}(w)=r B_{x-1, r}(w)+w B_{x-1, r+1}(w)
$$

where $x$ and $r$ are nonnegative integers. This will reduce to

$$
B_{x, r}=r B_{x-1, r}+B_{x-1, r+1}
$$

when $w=1$. Analogous to this relation, we have the following relation which can easily be shown using (28), (15), and (16).

Theorem 13. The r-Bell numbers satisfy the following relation:

$$
B_{x, r}=r B_{x-1, r}+B_{x-1, r+1}+\left\{\begin{array}{c}
x+r-1 \\
r-1
\end{array}\right\}_{r+1},
$$

where $x$ and $r$ are complex numbers.
Proof. Summing up both sides of (15) gives

$$
B_{x, r}=(r+1) B_{x-1, r}+\left\{\begin{array}{c}
x+r-1 \\
r-1
\end{array}\right\}_{r}+\sum_{k=0}^{\infty} k\left\{\begin{array}{c}
x+r-1 \\
k+r
\end{array}\right\}_{r} .
$$

By applying (16), we get

$$
\begin{aligned}
& \sum_{k=0}^{\infty} k\left\{\begin{array}{c}
x+r-1 \\
k+r
\end{array}\right\}_{r} \\
& =-\sum_{k=0}^{\infty}\left[\frac{1}{(k-1) !} \sum_{j=0}^{k}(-1)^{k-1-j}\left(\begin{array}{c}
k-1 \\
j
\end{array}\right)(\beta j+r)^{x-1}\right] \\
& \quad-\sum_{k=0}^{\infty}\left[\frac{-1}{(k-1) !} \sum_{j=0}^{k}(-1)^{k-1-(j-1)}\left(\begin{array}{c}
k-1 \\
j-1
\end{array}\right)((j-1)+1+r)^{x-1}\right] .
\end{aligned}
$$

Hence, we have

$$
\begin{aligned}
& \sum_{k=0}^{\infty} k\left\{\begin{array}{c}
x+r-1 \\
k+r
\end{array}\right\}_{r} \\
& \quad=-\left\{\begin{array}{c}
x+r-1 \\
r-1
\end{array}\right\}_{r}-B_{x-1, r}+B_{x-1,1+r}+\left\{\begin{array}{c}
x+r-1 \\
r-1
\end{array}\right\}_{r+1} .
\end{aligned}
$$

Substituting this to (35) completes the proof of the theorem.

Note that, when the parameters $x, y$, and $r$ are nonnegative integers, Definition 1 is just equivalent to the integral representation of the $r$-Stirling numbers of the second kind in [1]. Hence, the value of $\left\{\begin{array}{c}x+r-1 \\ r-1\end{array}\right\}_{r+1}$ is equal to 0 .

\section{Asymptotic Formula}

An asymptotic formula for $r$-Bell numbers $B_{x, r}$ was first established by Corcino in [7]. But the formula holds only when $x$ is a nonnegative integer. Here, we aim to obtain an asymptotic formula for $B_{x, r}$ when $x$ is a real number.

Using Definition 9,

$$
B_{x, r}=\frac{x !}{2 \pi i} \int_{\mathscr{H}} \frac{e^{r z+\left(e^{z}-1\right)}}{z^{x+1}} d z .
$$

To obtain an asymptotic formula, we deform the path $\mathscr{H}$ into the following contour: $C_{1} \cup C_{2} \cup C_{3} \cup C_{4} \cup C_{5}$, where

(i) $C_{1}$ is the line $\mathbb{I} z=-2 \pi+\delta, \delta>0$, and $\mathbb{R} z \leq \lambda, \lambda$ is a small positive number;

(ii) $C_{2}$ is the line segment $\mathbb{R} z=\epsilon$, going from $\lambda+i(\delta-2 \pi)$ to the circle $|z|=R$;

(iii) $C_{5}$ and $C_{4}$ are the reflections in the real axis of $C_{1}$ and $\mathrm{C}_{2}$, respectively;

(iv) $C_{3}$ is the portion of the circle $|z|=R$, meeting $C_{2}$ and $\mathrm{C}_{4}$. 
The new contour is $C_{1} \cup C_{2} \cup C_{3} \cup C_{4} \cup C_{5}$ in the counterclockwise sense. This idea of deforming the contour $\mathscr{H}$ is also done in [13]. The integrals along $C_{1}, C_{2}, C_{4}$, and $C_{5}$ are seen to be

$$
O\left(\frac{(2+\lambda)^{y}}{(2 \pi-\delta)^{x}}\right)
$$

It will also be shown that these integrals go to 0 as $x \rightarrow \infty$ provided that $x-y \geq x^{1 / 3}$. To see this, we consider $C_{1}$. For the other contours, the estimate can be seen similarly. We look at

$$
\left|\int_{C_{1}} \frac{e^{r z+\left(e^{z}-1\right)}}{z^{x+1}} d z\right| \leq \int_{C_{1}}\left|\frac{e^{r z+\left(e^{z}-1\right)}}{z^{x+1}}\right| d z \mid .
$$

Note that

$$
\begin{aligned}
\left|e^{r z+\left(e^{z}-1\right)}\right| & =\left|e^{r z}\right|\left|e^{\left(e^{z}-1\right)}\right| \\
& =e^{r \mathbb{R} z} e^{\mathbb{R}\left(e^{z}-1\right)} .
\end{aligned}
$$

Turning to $e^{z}-1$

$$
\begin{aligned}
e^{z}-1 & =e^{\mathbb{R} z} e^{i \mathbb{J} z}-1 \\
& =\left[e^{\mathbb{R} z} \cos \mathbb{I} z-1\right]+i e^{\mathbb{R} z} \sin \mathbb{I} z .
\end{aligned}
$$

Thus,

$$
\begin{aligned}
\left|e^{r z+\left(e^{z}-1\right)}\right| & =e^{r \mathbb{R} z} e^{e^{\mathbb{R} z} \cos \mathbb{J} z} e^{-1} \\
& <e^{r \mathbb{R} z} e^{e^{\mathbb{R} z}} e^{-1} .
\end{aligned}
$$

Consider

$$
\begin{aligned}
r \mathbb{R} z+e^{\mathbb{R} z}-1 & <r \lambda+e^{\lambda}-1 \\
& =r \lambda+\lambda\left(1+\frac{\lambda}{2 !}+\frac{\lambda^{2}}{3 !}+\frac{\lambda^{3}}{4 !}+\cdots\right) \\
& <r \lambda+\lambda\left(1+\frac{\lambda}{2}+\frac{\lambda^{2}}{2^{2}}+\frac{\lambda^{3}}{2^{3}}+\cdots\right) \\
& =r \lambda+\lambda\left(\frac{2}{2-\lambda}\right) .
\end{aligned}
$$

Choose $\lambda<1$, so that $2-\lambda>1$. Then

$$
r \mathbb{R} z+e^{\mathbb{R} z}-1<\lambda(2+r) .
$$

This implies that

$$
\left|e^{r z+\left(e^{z}-1\right)}\right|<e^{\lambda(2+r)}
$$

Consequently, we obtain

$$
\left|\int_{C_{1}} \frac{e^{r z+\left(e^{z}-1\right)}}{z^{x+1}} d z\right|<e^{\lambda(2+r)} \frac{1}{(2 \pi-\delta)^{x+1}} l\left(C_{1}\right)
$$

where

$$
l\left(C_{1}\right)=\lim _{t \rightarrow-\infty} \lambda-t
$$

Since $l\left(C_{1}\right) /(2 \pi-\delta)^{x+1} \rightarrow 0$ as $x \rightarrow \infty$, we have the integral along $C_{1}$ goes to 0 as $x \rightarrow \infty$. So what remains is the integral along $C_{3}$. That is,

$$
B_{x, r} \sim \frac{x !}{2 \pi i} \int_{C_{3}} \frac{e^{r z+\left(e^{z}-1\right)}}{z^{x+1}} d z,
$$

where $C_{3}$ is a semicircle $z=R e^{i \theta},-\alpha \leq \theta \leq \alpha$. Hence, by Laplace method or following the analysis in [7],

$$
\begin{aligned}
B_{x, r} & \sim \frac{x !}{2 \pi i R^{n}} \int_{-\alpha}^{\alpha} \exp \left(e^{R e^{i \theta}}+r R e^{i \theta}-i n \theta\right) d \theta \\
& \sim \frac{x !}{2 \pi i R^{n}} \int_{-\epsilon}^{\epsilon} \exp \left(e^{R e^{i \theta}}+r R e^{i \theta}-i n \theta\right) d \theta .
\end{aligned}
$$

In [7], the integration is along a circle about zero with radius $R$. This number $R$ is shown to be the unique solution to

$$
\frac{\mu}{x-r \mu}=e^{-\mu}
$$

as a function of $\mu$ (see Lemma 3 [7]). We see that the asymptotic formula for the $r$-Bell numbers obtained in [7] holds for real argument $x$. Thus, we have the following asymptotic formula.

Theorem 14. The $r$-Bell numbers $B_{x, r}$ with real arguments $x$ and $r$ have the following asymptotic formula:

$$
B_{x, r} \sim C \sqrt{\pi}\left[1+\frac{D-\left(2 R^{4}+9 R^{3}+16 R^{2}+6 R+2\right)}{24 e^{R} R\left(R+1+r e^{-R}\right)^{3}}\right],
$$

where

$$
\begin{aligned}
& C=\frac{x ! \exp \left(r R+e^{R}-1\right)}{\pi R^{x}\left[2\left(r^{2} e^{R}+R e^{R}+r R\right)\right]^{1 / 2}}, \\
& D=\left(3 R^{3}+8 R^{2}-6 R-4-2 r e^{-R}\right) r e^{-R},
\end{aligned}
$$

and $R$ is the unique positive solution to

$$
\mu e^{\mu}+r \mu-x=0
$$

as a function of $\mu$.

An asymptotic formula for $(r, \beta)$-Bell numbers $G_{x, r, \beta}$ has already been established in [14]. However, the formula holds only when $x$ is a nonnegative integer. Here, using the same method as employed above, we can show that this asymptotic formula will also work for the case in which the parameters $x, r$, and $\beta$ are real numbers.

Now, for real parameters $x, r$, and $\beta$, we have

$$
G_{x, r, \beta}=\frac{x !}{2 \pi i} \int_{\mathscr{H}} e^{r z+\left(e^{\beta z}-1\right) / \beta} \frac{d z}{z^{x+1}},
$$

where the path $\mathscr{H}$ can also be deformed into the following contour: $C_{1} \cup C_{2} \cup C_{3} \cup C_{4} \cup C_{5}$, such that

(i) $C_{1}$ is the line $\mathbb{I} z=-2 \pi+\bar{\delta}, \bar{\delta}>0$, and $\mathbb{R} z \leq \bar{\lambda}, \bar{\lambda}$ is a small positive number; 
(ii) $C_{2}$ is the line segment $\mathbb{R} z=\bar{\lambda}$, going from $\bar{\lambda}+i(\bar{\delta}-$ $2 \pi$ ) to the circle $|z|=\bar{R}$;

(iii) $C_{5}$ and $C_{4}$ are the reflections in the real axis of $C_{1}$ and $\mathrm{C}_{2}$, respectively;

(iv) $C_{3}$ is the portion of the circle $|z|=\bar{R}$, meeting $C_{2}$ and $\mathrm{C}_{4}$.

Also, the new contour is $C_{1} \cup C_{2} \cup C_{3} \cup C_{4} \cup C_{5}$ in the counterclockwise sense. It can easily be shown that, along the path $C_{1}$,

$$
\left|e^{r z+\left(e^{\beta z}-1\right) / \beta}\right|<e^{\bar{\lambda}((2 / \beta)+r)} .
$$

Hence, as $x$ goes to $\infty$,

$$
\left|\int_{C_{1}} e^{r z+\left(e^{\beta z}-1\right) / \beta} \frac{d z}{z^{x+1}}\right| \longrightarrow 0 .
$$

This can be done similarly along $C_{2}, C_{4}$, and $C_{5}$. Thus, we have

$$
G_{x, r, \beta} \sim \frac{x !}{2 \pi i} \int_{C_{3}} \frac{e^{r z+\left(e^{\beta z}-1\right) / \beta}}{z^{x+1}} d z,
$$

where $C_{3}$ is a semicircle $z=\bar{R} e^{i \theta},-\bar{\alpha} \leq \theta \leq \bar{\alpha}$. Then

$$
\begin{aligned}
G_{x, r, \beta} & \sim \frac{x !}{2 \pi i \bar{R}^{n}} \int_{-\bar{\alpha}}^{\bar{\alpha}} \exp \left(\beta^{-1} e^{\beta \bar{R} e^{i \theta}}+r \bar{R} e^{i \theta}-i x \theta-\beta^{-1}\right) d \theta \\
& \sim \frac{x !}{2 \pi i \bar{R}^{n}} \int_{-\epsilon}^{\epsilon} \exp \left(\beta^{-1} e^{\beta \bar{R} e^{i \theta}}+r \bar{R} e^{i \theta}-i x \theta-\beta^{-1}\right) d \theta .
\end{aligned}
$$

This implies that the asymptotic formula for the $(r, \beta)$-Bell numbers obtained in [14] holds for real argument. Thus, from [14], we have the following asymptotic formula for $(r, \beta)$-Bell numbers $G_{x, r, \beta}$ with real arguments $x, r$, and $\beta$ :

$$
\begin{aligned}
& G_{x, r, \beta} \\
& \sim \frac{x^{1 / 2}(1+1 / 12 x) \exp \left(r \bar{R}+\beta^{-1} e^{\beta \bar{R}}-\beta-x\right)\left(\beta^{\beta \bar{R}}+r\right)^{x}}{\left[(x-r \bar{R}) \beta^{-1}\right]^{1 / 2}\left(\beta \bar{R}+1+r e^{-\beta \bar{R}}\right)^{1 / 2}} \\
& \quad \times\left(1+\frac{D+E}{F}\right),
\end{aligned}
$$

where

$$
\begin{aligned}
D= & \left(3 \beta^{2} \bar{R}^{3}+8 \beta \bar{R}^{3}+3 \beta \bar{R}+3-10 \beta^{-1}-2 r e^{-\beta \bar{R}}\right) r e^{-\beta \bar{R}}, \\
E= & \left(3 \beta^{3}-5 \beta^{2}\right) \bar{R}^{4}+\left(21 \beta^{2}-30 \beta\right) \bar{R}^{3}+(39 \beta-55) \bar{R}^{2} \\
& +\left(24-30 \beta^{-1}\right) \bar{R}+\left(3 \beta^{-1}-5 \beta^{-2}\right), \\
F= & 24 \bar{R} e^{\beta \bar{R}}\left(\beta \bar{R}+1+r e^{-\beta \bar{R}}\right)^{3},
\end{aligned}
$$

and $\bar{R}$ is the unique positive solution to

$$
\mu e^{\beta \mu}+r \mu-x=0
$$

as a function of $\mu$.

\section{Summary and Recommendation}

In this paper, we have defined $r$-Stirling numbers of the second kind and $r$-Bell numbers with complex arguments using the concept of Hankel contour and established some properties parallel to those of the classical Stirling and Bell numbers. Moreover, we have derived an asymptotic formula for $r$-Bell numbers as well as for $(r, \beta)$-Bell numbers for real arguments using the method of Chelluri and that of Moser and Wyman.

We observe that, by employing those methods, one can possibly establish an asymptotic formula for $r$-Stirling numbers of the second kind with real arguments and, consequently, using relation (6), an asymptotic formula for $(r, \beta)$ Stirling numbers with real arguments.

\section{Acknowledgments}

The authors would like to acknowledge the support from the Office of the Vice Chancellor for Research and Development of the University of the Philippines Diliman for this research project. The authors also wish to thank the referees for reading and evaluating the paper thoroughly.

\section{References}

[1] A. Z. Broder, “The $r$-Stirling numbers," Discrete Mathematics, vol. 49, no. 3, pp. 241-259, 1984.

[2] I. Mezö, "On the maximum of $r$-Stirling numbers," Advances in Applied Mathematics, vol. 41, no. 3, pp. 293-306, 2008.

[3] I. Mezö, "New properties of $r$-Stirling series," Acta Mathematica Hungarica, vol. 119, no. 4, pp. 341-358, 2008.

[4] R. B. Corcino, C. B. Corcino, and R. Aldema, "Asymptotic normality of the $r, \beta$-Stirling numbers," Ars Combinatoria, vol. 81, pp. 81-96, 2006.

[5] I. Mezö, "A new formula for the Bernoulli polynomials," Results in Mathematics, vol. 58, no. 3-4, pp. 329-335, 2010.

[6] A. Ruciński and B. Voigt, "A local limit theorem for generalized Stirling numbers," Revue Roumaine de Mathématiques Pures et Appliquées, vol. 35, no. 2, pp. 161-172, 1990.

[7] C. B. Corcino, "An asymptotic formula for the $r$-Bell numbers," Matimyás Matematika, vol. 24, no. 1, pp. 9-18, 2001.

[8] I. Mező, “The $r$-Bell numbers," Journal of Integer Sequences, vol. 14, no. 1, article 11.1.1, 2011.

[9] R. B. Corcino, M. B. Montero, and C. B. Corcino, "On generalized Bell numbers for complex argument," Utilitas Mathematica, vol. 88, pp. 267-279, 2012.

[10] L. Moser and M. Wyman, "An asymptotic formula for the Bell numbers," vol. 49, pp. 49-54, 1955.

[11] R. L. Graham, D. E. Knuth, and O. Patashnik, Concrete Mathematics, Addison-Wesley, Reading, Mass, USA, 2nd edition, 1994.

[12] P. Flajolet and H. Prodinger, "On Stirling numbers for complex arguments and Hankel contours," SIAM Journal on Discrete Mathematics, vol. 12, no. 2, pp. 155-159, 1999.

[13] R. Chelluri, L. B. Richmond, and N. M. Temme, "Asymptotic estimates for generalized Stirling numbers," Analysis, vol. 20, no. 1, pp. 1-13, 2000. 
[14] R. B. Corcino and C. B. Corcino, "On generalized Bell polynomials," Discrete Dynamics in Nature and Society, vol. 2011, Article ID 623456, 21 pages, 2011. 


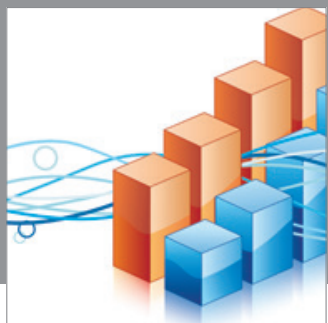

Advances in

Operations Research

mansans

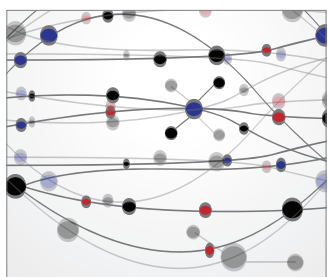

The Scientific World Journal
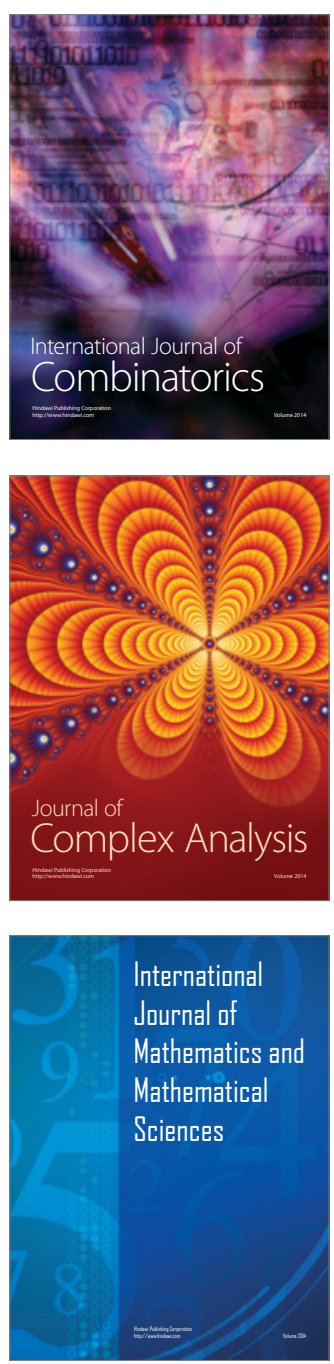
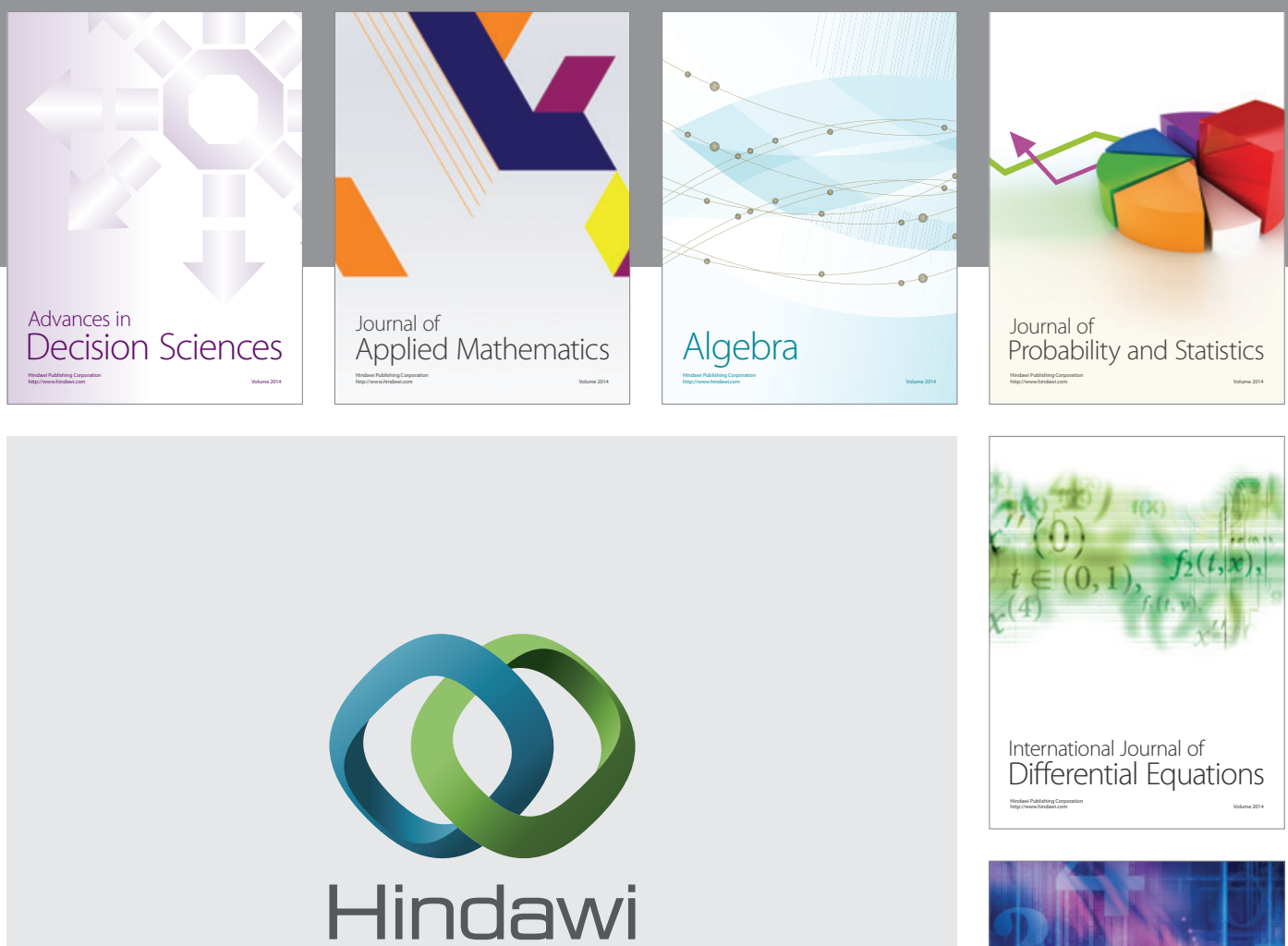

Submit your manuscripts at http://www.hindawi.com
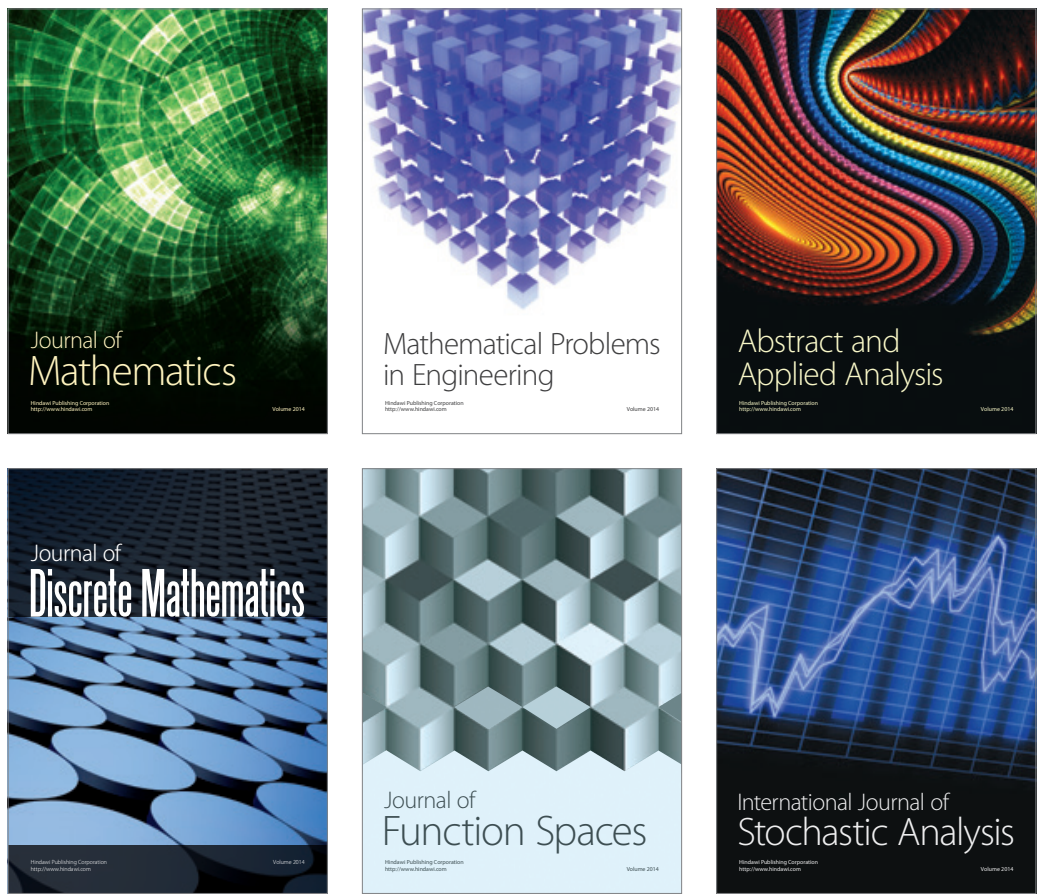

Journal of

Function Spaces

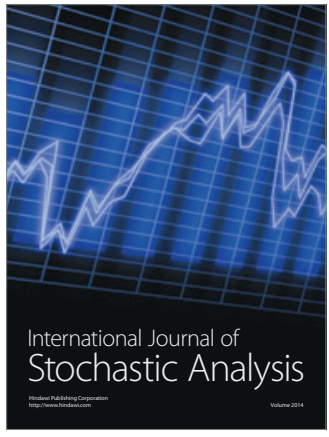

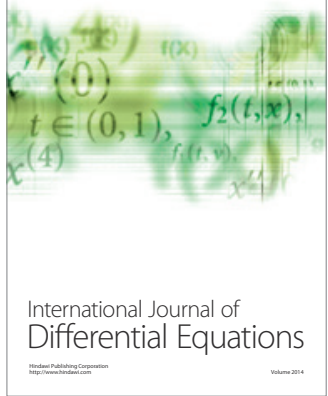
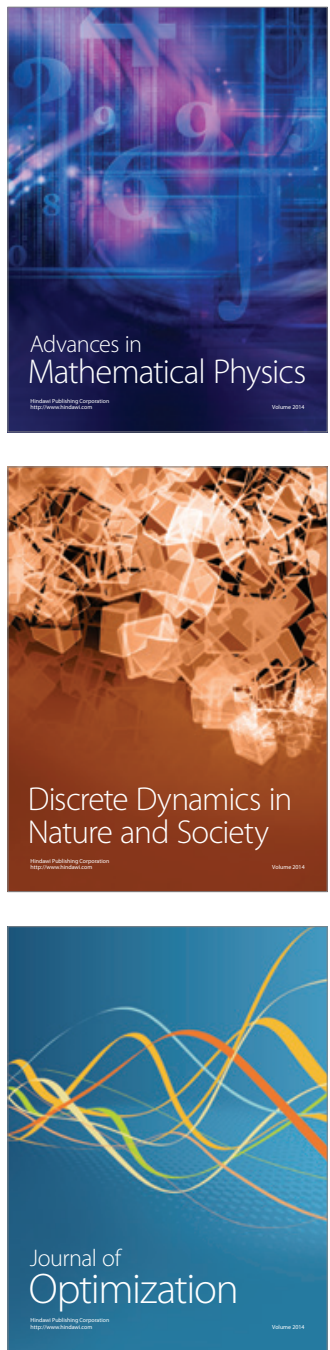